\title{
ATLA \& PALNI OCLC WMS Pre-Conference
}

Panel Discussion: Knowledge Base Management of Open Access Resources

Lisa Gonzalez, PALNI

Jeff Siemon, Anderson University

Heather Loehr, PALNI

Karl Stutzman, Anabaptist Mennonite Biblical Seminary

\section{Focusing on Open Access in WMS}

Lisa Gonzalez, PALNI

Considering that open access (OA) collections contributed by both vendors and other libraries represent significant financial investment by institutions through either APCs (article processing charges), membership support, or other investment in the scholarly communication infrastructure, libraries should evaluate OA collections that match their collection development policy subject priorities. Since OA material may be aggregated inconsistently in diverse locations, adding OA content to the library's discovery system provides another avenue for patrons to discover quality content.

You can select OA collections broadly through WorldCat Discovery or Collection Manager settings, or more granularly one collection at a time or even title-by-title. The basic information about OA collections on OCLC's knowledge base collections list includes the size of the collection and the percentage of OCLC numbers that are linked to titles in the knowledge base. OCLC numbers connect e-resource title holdings to WorldCat records and to article citations in WorldCat.org and WorldCat Discovery based on the library's coverage for titles.

When evaluating OA collections in the knowledge base, selection criteria can include whether the collection is sufficiently focused on material relevant to your patrons, the quality of the metadata in terms 
of accurate description (title, URLs, identifiers), and if the material is actually available open access. When contributing OA collections to the knowledge base, consider providing the most complete metadata possible (full titles, ISBNs, OCLC numbers, accurate URLs) before submitting the collection to be shared. Tools such as the MarcEdit KBART plugin can help you quickly create a KBART file for your knowledge base collection, which you can check for accuracy and completeness before you create your collection in the knowledge base.

\section{Tips for Fixing OCLC Knowledge Base Broken Links}

Jeff Siemon, Anderson University

We'll address the problem that patrons cannot find e-resources we provide, and have paid for, because many e-resources have poor metadata. Yukari Sugiyama writes, and I agree:

E-resource discovery is almost entirely dependent upon metadata that is supplied by parties outside of the library. ... Libraries now work in an environment where there are more interdependencies than ever; the successful provision of access to electronic resources is fully dependent on the transmission of high quality metadata throughout the e-resource supply chain.

We will cover some ways to improve OCLC knowledge base collections:

- Add subtitles to prevent incorrect matches in Discovery to books with similar titles (many are HathiTrust short titles).

- Add a primary=override OCLC Number (OCN) to knowledge base titles without primary OCNs.

- Change (override) a print book primary OCLC number with the best eBook OCN.

- Add open access collections to your catalog. 
- Add free or open access journals not in any KB collection to the collection: "Other Free Journals" ID:freeAccess.misc.

One common problem is that OCLC Discovery links to a wrong book with the same title. The solution is as follows:

1. In Discovery, follow the (wrong) link to the eBook in HathiTrust. Get the number from the URL of the (wrong) linking eBook (e.g., 4287499), and note the subtitle or series title for that book.

2. In Collection Manager, search for your HathiTrust KB collection. Then search for the HathiTrust number, 4287499, in that collection.

3. Add a subtitle or series title to KB title. (You can add an author to the title if you have no other choice.)

4. Save your changes (blue "Save" button).

Then contribute your changes to the knowledge base:

5. Click the drop-down box "Contribute Changes to the Global Collection."

6. Select "Add title edits to the Global Knowledge Base."

7. In "summary of change" box, type something simple like "added subtitle."

8. Click "Continue."

The result is that the wrong "Access online" link is gone from the 1990 book with a similar title.

But there is "fine print" to this simple solution to fixing wrong links to similarly titled books. You will need to repeat these steps for each wrong HathiTrust link appearing in Discovery with the (usually more recent) book.

Also, I delete most of the grouped OCNs in the HathiTrust knowledge base record, leaving only a few of the OCNs for the most held correct print and eBook WorldCat records. It would be nice to check every OCN on the HathiTrust KB title record, but perfection takes too much time. The pros for deleting a wrong OCN outweigh the cons for deleting an extra correct OCN. Most of the correctly grouped OCNs will also be clustered in Discovery; thus, even when you delete a correct OCN from the KB record, the "View eBook" link will still appear on the cluster in Discovery. When you delete (wrong) group OCNs, you will 
have fixed "broken links" issues for other similar titles. Discovery is improved by adding OCLC numbers to KB collections.

A second common problem is that there is no "override" or primary OCLC number for a title in the OCLC knowledge base. When there is no primary OCN in the KB, then holdings are not set in WorldCat for libraries that use WMS for their catalog. And for non-WMS libraries, no MARC record is delivered to be loaded in their catalog. Therefore, that title shows up lower in search results, and that title will not display if the search is limited to My Library Holdings. The "Access online" link may still show up on a result, if it matches ISBN/ISSN or title, or if the OCN is in the KB in the "grouped OCNs" list.

The second common problem is also easy to fix by adding an "override" OCN to the OCLC knowledge base:

1. Search WorldCat for the book/journal that has no OCN in the knowledge base and copy the OCLC number to the clipboard.

2. Go back to the knowledge base and paste the OCN in the "override" OCN field.

3. Save the record, and "Contribute Changes to the Global Collection" as above.

These titles that you've improved with OCLC numbers in the knowledge base can now be discovered by and delivered to your patrons!

A third common problem is print book MARC record OCLC numbers that are the "override" or primary OCN in the knowledge base. It looks like your library holds the print book, but you only hold the eBook. To correct a print OCN as the primary OCN in the KB:

1. Find the eBook title in the knowledge base; perhaps search by the (wrong) print OCN. Begin to edit that KB record.

2. Search in WorldCat for an eBook MARC record for that book, and copy the eBook OCN.

3. Back in Collection Manager, in the KB record, paste the eBook OCN in the "override" OCN field.

4. Copy the current primary OCN, add it to the "grouped" OCNs, and save that OCN.

5. Save the entire KB title record.

6. Contribute the title edits to the global knowledge base. 
A fourth easy task to help your patrons is to add open access e-resources collections to your library catalog. Here's how:

You can find open access collections in Collection Manager by searching for the word "open" or searching all collections and limiting by the OA facet. A good place to start for seminaries is by searching for "DTL Open." This retrieves open access collections created by the Digital Theological Library, a project of Claremont.

All you need to do is click on the "Select" button near the right side of the results list, and these open access titles will show up in your WMS catalog. If you use another vendor, the MARC records for these titles will be delivered for you to load into your local catalog.

In addition to these four simple tasks, Jeff also showed slides about how to add a new open access journal to a knowledge base collection and how to add OCLC number to a collection using a KBART file.

Slides from this presentation may be found at: https://www. slideshare.net/jsiemon/presentations

Recording: https://vimeo.com/237468207/00de177b16

Detailed instructions for adding multiple OCNs to an existing knowledge base collection may be found at: https://drive. google.com/open?id=0B2sHKamxnI-dQmFkcUVmS1d4RTQ (about 50 pages).

A checklist for what to do when adding or canceling an e-resource or a database may be found at: https://docs.google. com/document/d/1yuf4g-aRA6Fh7eJno4GtSryjEkI22gycalxhzdAHmsY/edit?usp=sharing.

\section{Panel Discussion: Reporting and Statistics}

Heather Loehr, PALNI

Karl Stutzman, Anabaptist Mennonite Biblical Seminary

We discussed a brief history of the PALNI national survey support project, a collaborative effort between PALNI librarians to provide instruction and guidance on using WMS analytics and reports for data-gathering for the ACRL, IPEDS, and ATS surveys. 
We showed the common data points required by each survey and the available methods for addressing each one. Gathering needed data for national surveys presents a number of challenges for WMS libraries-including unclear survey instructions, limitations of standard ("out of the box") reporting methods in WMS, and limitations of data universes for WMS Report Designer customers. We presented the first working draft for a help guide for the ATS survey, and we provided an overview of enhancement requests submitted to OCLC for system features and functions that can improve the efficiency of data-gathering, and the quality of the data gathered, to align as closely as possible with the needs of reporting libraries.

Slides from this presentation may be found at: http://

libguides.palni.edu/ld.php?content_id=44679130.

PALNI's National Survey Guides and Tutorials: http://

libguides.palni.edu/analytics/surveys

\section{NOTES}

1 Yukari Sugiyama, Marlene van Ballegooie, and Fabiano Takashi Rocha, "Harnessing the Power of the Cooperative: Improving Access to the JapanKnowledge E-Resource Collection," Journal of East Asian Libraries no. 163 (October 2016). 